\title{
Soft tissue surgery as an initial treatment for hip displacement in spastic cerebral palsy
}

\author{
Luiz Antônio Angelo da Silva ${ }^{1}$, and Patricia Maria de Moraes Barros Fucs ${ }^{2, *}$ \\ 1 Santa Casa Medical School and Hospitals, R. Dr. Cesário Mota Junior 61, 01221-020 São Paulo/SP, Brazil \\ 2 Department of Orthopaedic and Traumatology, Irmandade da Santa Casa de São Paulo, Santa Casa Medical School and Hospitals, \\ R. Dr. Cesário Mota Junior 61, 01221-020 São Paulo/SP, Brazil
}

Received 17 August 2020, Accepted 30 August 2020, Published online 12 October 2020

\begin{abstract}
Objective: To use the measurement of migration percentage (MP) to evaluate the long-term radiographic results of soft tissue surgery as the first treatment for hip displacement in spastic bilateral cerebral palsy. A secondary objective was to identify predictive factors of stability (i.e., less than $30 \%$ of MP in the long term), after surgical correction. Methods: In this longitudinal cohort study, we reviewed the electronic medical records and radiographs of all consecutive patients with cerebral palsy operated for the correction of hip displacement between 1984 and 2013 in a referral orthopedic public hospital in Brazil. Patients were included if they had received, as the first surgical procedure, soft-tissue release. All surgeries were bilateral and symmetrical. We used the available radiographs to evaluate the migration percentage (MP), acetabular index (AI), pelvic obliquity (PO) angle, head-shaft angle (HSA), congruence and femoral head sphericity, and function using the GMFCS (Gross Motor Function Classification System). Results: we included 93 patients, all operated before being 12 years old, with follow-up of 10 years in average, $73(78 \%)$ of them with good results (MP $<30 \%)$. We found association between preoperative MP $\leq 40 \%$, $\mathrm{AI} \leq 25^{\circ}$, and postoperative symmetry with good results, with a cut-off value of $38 \%$ of MP and $27^{\circ}$ of acetabular index being predictive. Discussion: The role of soft tissue releases remains controversial owing to small sample sizes, heterogeneity, variety range of ages, definitions used for outcome, and lack of statistical quality. Our results were better in combined tenotomies, in diparetic patients aged more than six years, and in patients with lower initial values of MP and AI. Radiographic variables had good correlation with each other and association with results, with cut-off values for MP and AI PRE.
\end{abstract}

Key words: Cerebral pasly, Hip subluxation, Tenotomy, Muscle spasticity.

\section{Introduction}

The incidence of hip dislocation has been reported in $2.6-34 \%$ of children with cerebral palsy, resulting in pain and in quality-of-life reduction [1]. The basis for surgical treatment of hip dislocation in these cases is obtaining balance of the pelvis. However, there is still no consensus on which muscles should be addressed, the ideal age, the degree of dislocation that can be successfully treated [2], the post-operative care, the ways to evaluate the results and the factors that influence the results of soft tissue release.

Early detection of hip displacement is advocated, but it is not safe through clinical examination alone or by evaluating other risk factors [3]. It is necessary to perform radiographs.
However, different limit values have been adopted as radiographic parameters in the literature, depending on the objective of the treatment and the type of evaluation. In addition, there are problems regarding accuracy, confidence, and inadequate patient positioning for radiographic assessments [2, 3]. The available studies are retrospective cohorts with patients of different age groups, radiographic and functional preoperative statuses and categories, length of follow-up, clinical subtypes, and evaluation criteria, leading to results that are difficult to compare [4]. Moreover, few studies have been published with a long follow-up time [5-7].

The main objective of this research is to use the migration percentage (MP) to evaluate the long-term radiographic results of soft tissue surgery as the first treatment for hip displacement

*Corresponding author: patricia.fucs@fcmsantacasasp.edu.br

This is an Open Access article distributed under the terms of the Creative Commons Attribution License (https://creativecommons.org/licenses/by/4.0), which permits unrestricted use, distribution, and reproduction in any medium, provided the original work is properly cited. 
in spastic bilateral cerebral palsy. The secondary objective is to identify predictive factors of stability (i.e., less than $30 \%$ of MP in the long term), after surgical correction.

\section{Methods}

\section{Study design, setting, and ethics}

This is a cross-sectional study, based on the review of electronic medical records and radiographs of all consecutive patients with cerebral palsy operated for the correction of hip displacement between 1984 and 2013 in a referral orthopedic public hospital in Brazil. This study was carried out between 2019 and 2020 after approval of the institutional review board (protocol 3.597.206). As the investigation was based on medical records review, no informed consent could be obtained specifically for this study, but all parents and caretakers consented for the medical procedures and were informed about the possibility of use of aggregated data from anonymized records.

\section{Participants}

We reviewed the hospital electronic medical records in search of cases of children with spastic bilateral cerebral palsy who received, as the first surgical procedure, soft-tissue release. We used the following inclusion criteria:

- age of 12 years or less at the first surgery;

- available preoperative and postoperative radiographs, with anterior-posterior view of the hip;

- at least one hip with migration percentage (MP) of less than $90 \%$;

- data in the medical record comprising at least four years of follow-up after surgery.

All surgeries included in this study were bilateral, as per the departmental routine, and symmetrical. They were conducted starting with a medial incision over the long adductor muscle to access the set of adductors, indicated by the clinical test showing the abduction limitation $<30^{\circ}$. The most proximal tenotomy possible was performed by the same route in case of hip flexion contracture $>30^{\circ}$, and hamstrings were released distally in case of shortening on physical examination.

\section{Variables, measurements and bias reduction}

We used the available radiographs to evaluate the migration percentage (MP) and also to perform the following set of additional measurements:

- Acetabular index (AI);

- Pelvic obliquity (PO) angle;

- Head-shaft angle (HSA).

For these measurements, we used the ImageJ processor (National Institutes of Health) over the digital radiographs.

To avoid bias, the same evaluator took these measurements twice (20), aiming to get the same result. If there was difficulty

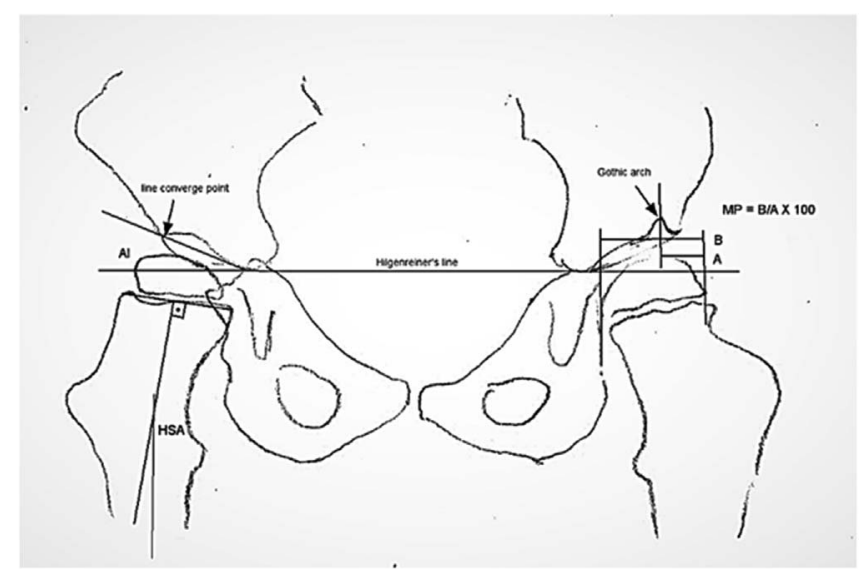

Figure 1. References for the calculation of migration percentage (MP) in the presence of a Gothic arch, acetabular index (AI) in the case of oval image, and the head-shaft angle (HSA).

in choosing reference points in the images for the lines drawing, some measurements were not taken. We could not evaluate AI in $6 \%$ of the images, where there was extreme pelvis rotation. We could not evaluate HAS in cases of great exposure of the small trochanter or when the femur had a "scoliotic" aspect.

In the presence of a Gothic arch, we drew a Perkins' line at the apex of the arch; if there was pelvic anteversion, we used a bisector of the oval image by double acetabular lines (Figure 1). We did not measure AI or HSA in cases with extreme rotations, because it could compromise accuracy.

We verified in the radiographs if the postoperative MP was less than $30 \%$. In these cases, and if the patient was not submitted to osteotomy as a revision surgery, the result was registered as good. If the patient underwent soft tissue release, but MP was still less than $30 \%$, the result was also good.

We also evaluated, in the postoperative radiographs, the morphology of the hips for congruence and femoral head sphericity.

Besides demographic data, we collected the functional evaluations from the medical records, which were performed at the time using the GMFCS (Gross Motor Function Classification System).

\section{Statistical analysis and study size}

This study used a convenience sample comprising all medical records available since inception of the electronic medical records in the hospital, with no previous sample size calculation.

We described data as frequencies and averages. We used chi-squared or Fisher exact test as appropriate for qualitative and Student's $t$ test or Mann-Whitney for quantitative variables to examine the association between them and good results. We calculated the correlation between quantitative variables using scatter plots and Pearson's or Spearman's correlation analysis. We used Epi-Info 7.2 and SPSS 13.0 for receiver operator characteristic (ROC) curves. We adopted $p$-values $<0.05$ as significant. 


\section{Results}

In the study period, 93 patients were operated and included in the study, $57 \%$ boys. They had all been operated before being 12 years old, in average at 6.8 years (standard deviation [SD] = 3 years; range: $2.4-12$ years). The mean follow-up period was 10.3 years $(\mathrm{SD}=6)$ years, most $(73 \%)$ with follow-up longer than 6 years, and 54\% reaching skeletal maturity at the last follow-up. The age at the final evaluation was in average 17 years. All cases had digital radiographs available for the PRE and final evaluations.

Among all patients, 73 (78\%) were considered to have good results. For 63 patients (68\%) with subluxated hips initially (MP > 25\%), the result was considered good. For all the 30 patients with hips "at risk", the result (MP of $25 \%$ or less) was good. There was no significant difference of age between patients considered as having good or bad results. We found MP PRE $>40 \%$ associated with the age of 4 years and MP PRE $\leq 40 \%$ aged 7 years with statistical significance.

Most patients were diparetic $(65 \%)$, and $90 \%$ of these cases were associated with good results $(p<0.001)$. The functional level was available for 80 patients, showing $65 \%$ of them with GMFCS (Gross Motor Function Classification System) IV-V $(p=0.72)$. GMFCS remained unchanged at 59\%, $25 \%$ had functional worsening, and 19\% of patients had improvement in the follow-up (100\% associated with good results; $p=0.03$ ).

Combined tenotomies $(70 \%)$ had better results than the isolated ones $(p=0.03)$ especially those involving the adductor, psoas, and hamstrings $(16 \% ; p=0.02)$, or when they were combined with hamstring tenotomies $(46 \%) p=0.007$.

We evaluated the association of the mean MP, AI, HSA, PO measurements with the good or bad results (Table 1). We also evaluated the difference of MP between hips preoperatively and the association with good or bad results: preoperatively 8 and 17 , respectively $(p=0.02)$; postoperative 7 and 20, $(p=0.15)$; and final 5 and $21(p<0.001)$. The MP mean measurements (Figure 2) decreased from the preoperative to postoperative and final follow-up evaluations (30\%, 27\% and $22 \%$ respectively), and the same happened with AI $\left(25^{\circ}, 24^{\circ}\right.$ and $23^{\circ}$ ) and $\operatorname{HAS}\left(155^{\circ}, 154^{\circ}\right.$ and $\left.148^{\circ}\right)$.

Scatter plot shows strong positive correlation between MP and AI postoperatively (Figure 3), with 0.832 according to Spearman's linear regression, and final (0.786, Pearson). It shows also moderate correlations between MP and AI preoperatively (0.624, Pearson), and MP and HSA (0.535, Spearman), AI and HSA (0.495, Spearman) and MP and PO (0.475, Spearman) postoperatively.

Among all patients, $41 \%$ needed revision surgeries for bone or soft tissue. These had higher MP mean (36\%) compared with children not reoperated $(27 \% ; p=0.01)$. These cases were more frequently isolated adductor releases $(p=0.005)$.

Additional soft tissue was necessary for $32 \%$ of the patients, considered as adjustments. Bone corrections were performed in 10 of the 93 patients, for the hip with worst results: varus derotation osteotomy [2], pelvic and femoral osteotomy [8], salvage [1], bilateral [9]. The MP mean preoperatively was always $52 \%$ versus $28 \%$ for patients undergoing or not the bone revision surgery $(p<0.001)$.
Table 1. Preoperative (PRE), postoperative (POST) and final mean radiographical measurements and differences according to Student's $t$ or Mann-Whitney's tests.

\begin{tabular}{lcccccc}
\hline Variables & PRE & $p$ & POST & $p$ & Final & $p$ \\
\hline MP (Good) & $25 \%$ & $<0.001$ & $20 \%$ & $<0.001$ & $17 \%$ & $<0.001$ \\
MP (Bad) & $51 \%$ & & $49 \%$ & & $44 \%$ & \\
AI (Good) & $24^{\circ}$ & $<0.001$ & $21^{\circ}$ & $<0.001$ & $21^{\circ}$ & $<0.001$ \\
AI (Bad) & $31^{\circ}$ & & $34^{\circ}$ & & $31^{\circ}$ & \\
HSA (Good) & $154^{\circ}$ & 0.35 & $153^{\circ}$ & 0.10 & $151^{\circ}$ & 0.09 \\
HSA (Bad) & $156^{\circ}$ & & $158^{\circ}$ & & $144^{\circ}$ & \\
OP (Good) & $2^{\circ}$ & 0.28 & $3^{\circ}$ & 0.04 & $3^{\circ}$ & $<0.001$ \\
OP (Bad) & $3^{\circ}$ & & $5^{\circ}$ & & $8^{\circ}$ & \\
\hline
\end{tabular}

$\mathrm{MP}=$ migration percentage; $\mathrm{AI}=$ acetabular index HSA = headshaft angle; $\mathrm{PO}=$ pelvic obliquity angle.

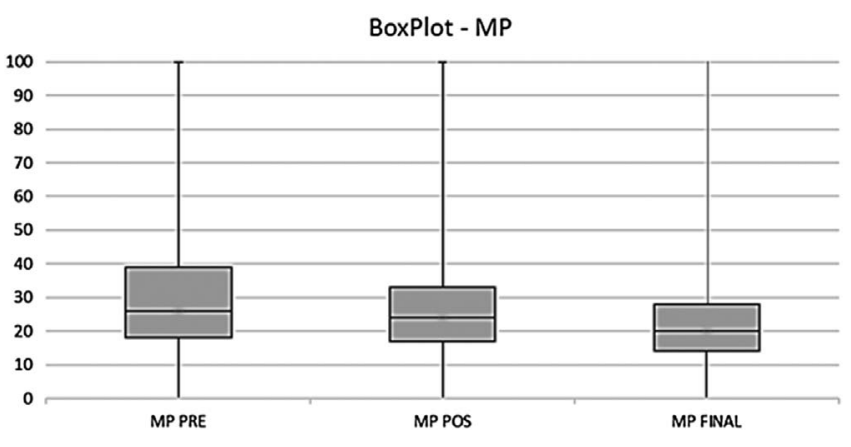

Figure 2. Preoperative (PRE), postoperative (POS), and final medians of migration percentage (MP): $26 \%, 24 \%$ and $20 \%$ respectively.

The morphology evaluation showed the following results for the worst hip of each patient: 81 were spherical and congruent, 2 spherical and incongruent, and 2 needed salvage. Among the 93 patients, 89 (96\%) had final congruency.

\section{Discussion}

The studies about spastic cerebral palsy in the literature tend to be based on heterogeneous groups, which makes their comparison and understanding difficult $[10,11]$. The inclusion criteria adopted in our study provided a homogeneous sample of children of the same age range, all with spastic bilateral cerebral palsy, and submitted to soft tissue surgery as the first procedure. This study showed that diparetic children $(p<0.001)$ and those operated after six years of age $(p=0.02)$ had better results, in contrast to findings in the literature with younger children [8]. Our study thus suggests that it is worthwhile to start the treatment with conservative approaches in this range age.

Adductor tenotomies, and surgeries involving the psoas and hamstrings (16\%), or those that associate hamstrings had better results compared to adductor surgery alone or with psoas, with statistical significance. Kalen and Bleck [1], found better results including the psoas than with isolated adductor tenotomies, and other authors include the psoas release frequently [6, 12].

We observed a migration of patients with GMFCS levels I-III to the IV-V level during follow-up, showing 


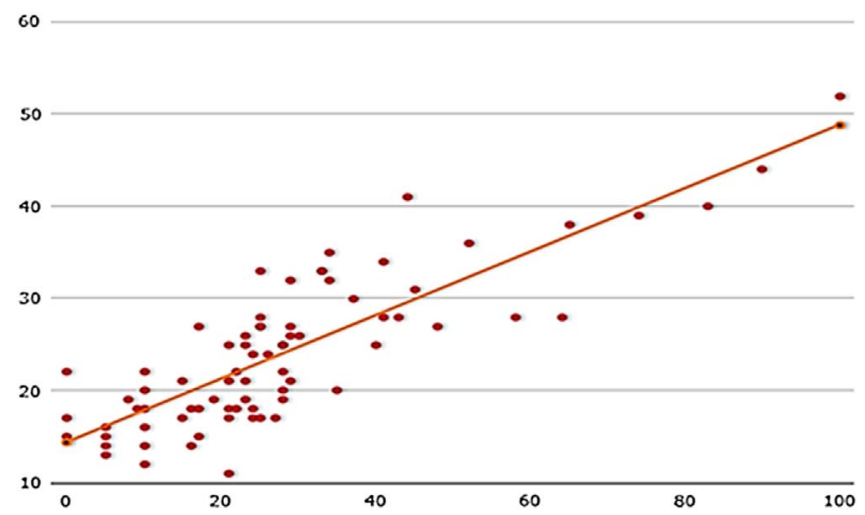

Figure 3. Distribution of migration percentage (MP) and acetabular index (AI) after surgery.

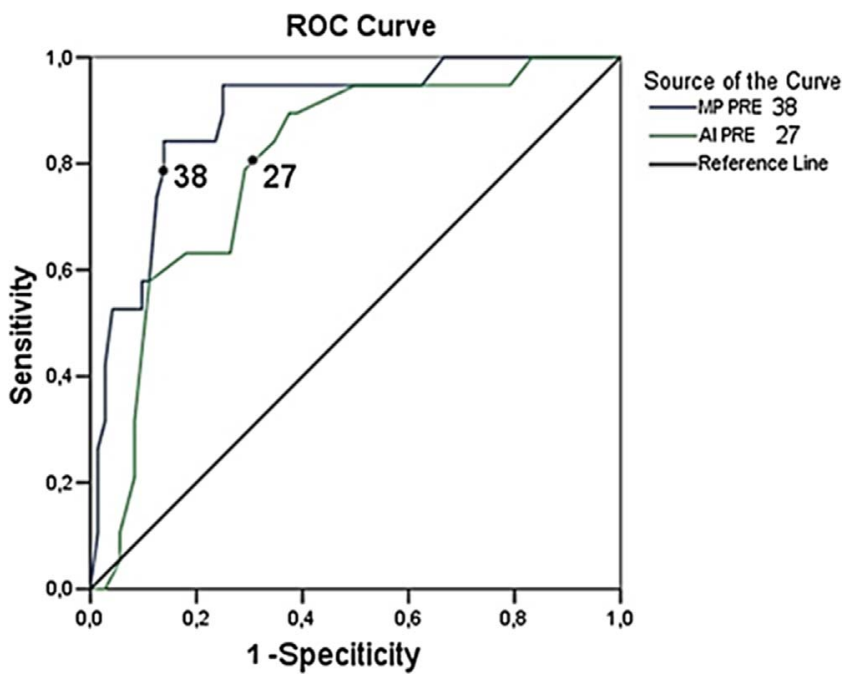

Figure 4. Receiver operator characteristic (ROC) curve with cut-off values of $38 \%$ (AUC 0.897) for migration percentage (MP), and $27^{\circ}$ (AUC 0.769) for acetabular index (AI), before (PRE) surgery.

functional worsening even with the treatment, but most patients' function remain unchanged (59\%). Predominant preoperative levels were IV-V $(65 \%)$.

According to other authors $[6,13,14]$ view, a second soft tissue surgery (that happened in $32 \%$ of the patients) was not considered a complication, and we did not exclude these patients from the analysis. Bone surgeries (a poor outcome criterion) were performed in the follow-up for ten patients. Revision surgeries (both for soft tissue or bone correction) were registered for 41 patients, and associated with higher MP preoperatively $(36 \% ; p=0.01)$ and with isolated adductors release, more than with combined surgeries $(p=0.005)$. In fact, other studies have evidenced the need for additional surgeries in $20-60 \%[4,5,14]$.

As already mentioned, we could not evaluate $\mathrm{AI}$ in $6 \%$ of the images, where there was extreme pelvis rotation, and HSA in cases of great exposure of the small trochanter or when the femur had a "scoliotic" aspect, as argued for by Tönnis [15]. However, this decision is unusual in other studies, where positioning and contractures have a great influence on measurements and results [9].

MP preoperative values were between $0 \%$ and $89 \%$, mostly below 39\% (Figure 2). We found evidence between age of 4 years old and MP $>40 \%$, in the same way 7 years old and MP $\leq 40 \%$ ( $p<0.001)$, showing a relationship between age and MP value in surgical indications similar to the indication profile of Presedo et al. [6].

Preoperative $25 \%$ and $51 \%$ MP mean were associated with good and bad results, respectively $(p<0.001)$, with average decreasing with treatment until final evaluation. Shore et al. [7] found preoperative MP averages of $21 \%$ and $45 \%$, and Turker and Lee [5] found $33 \%$ and $43 \%$ associated with success and failure, showing that the initial degree of subluxation can interfere with soft tissue surgery success $[5,7,16]$.

Mean AI decreased one point preoperatively, postoperatively and in the final evaluation (25-24-23 degrees), although other studies have not found improvement in AI after soft tissue procedures $[17,18]$. Preoperative AI $\leq 25 \%$ had $96 \%$ of hips associated with good, and preoperative averages $24^{\circ}$ versus $31^{\circ}$ associated with good and bad results, respectively $(p<0.001)$.

We evaluated the symmetry principle, based on the difference between MP values of the hips. Macias-Merlo et al. [19] found differences in the treated and control groups $10 \%$ and $35 \%$, respectively, with statistical significance. We infer that MP differences $\leq 10 \%$ between hips give rise to symmetrical hips, and larger differences to asymmetric hips. The exploration of the concept showed a linear improvement of the MP differences toward symmetry. Postoperative symmetry was associated with the results $(p=0.003)$.

The radiographic parameters had strong and moderate correlations with each other, and the ROC curves showed excellent preoperative cut-off values for MP $38 \%$ and AI $27^{\circ}$ (Figure 4). Cornell et al. [2] found MP $\geq 40 \%$ and $\mathrm{AI} \geq 27^{\circ}$, responding less to treatment [4].

Pelvic obliquity is often associated with bilateral cerebral palsy, influencing outcomes $[1,8]$. Averages of $3^{\circ}$ and $5^{\circ}$ postoperative were associated with good and bad results $(p=0.04)$, respectively in our study. Postoperative measurements were associated with the result, with statistical significance (Table 1).

We consider MP $\geq 30 \%$ as a clear abnormal result, that shows the subluxation is subject to progression in the child with severe involvement $[11,20]$. The overall result was good for 73 of 93 of our patients (78\%) comparable to that of another study using similar methodology [6].

For $63(68 \%)$ patients with initial subluxated hips (MP > 25\%) it was good, and for 30 patients with "hips at risk" (MP $\leq 25 \%$ ) it was $100 \%$ good shows the value of the initial MP as a predictive factor. Bowen and Kehl [12] consider it necessary to identify precisely which hips will subluxate without treatment (studies show 26-78\%), however, without more complete knowledge of the natural history of each hip, or the impossibility of close monitoring, most surgeons will intervene in smaller MPs, as in our study patients.

In the literature, success rates have been related to the duration of the follow-up, and high rates $(70 \%)$ are found in follow-ups of less than 5 years [4, 8]. Turker and Lee [5] claim that a follow-up of more than six years is necessary to 
determine the outcome of soft tissue surgery. A study found $73 \%$ of patients with a follow-up longer than six years and, although not all patients reached skeletal maturity, $89 \%$ were more than 10 years old in the final evaluation, older than the age when the highest incidence of dislocation occurs, although some patients suffer from luxation during the growth spurt [11]. Hips that are morphologically classified as $96 \%$ congruent at the final evaluation suggest that the patient will probably have a good evolution and favorable anatomy in future procedures that preserve the joint (tenotomies or reconstructive surgeries).

\section{Conclusions}

This study has shown that $78 \%$ of children with cerebral palsy and spastic hips have good results, evaluated by migration percentage in radiographs, after soft tissue release, not needing revision bone surgeries in the follow-up. Preoperative migration percentage of less than $40 \%$ and acetabular index $\leq 25^{\circ}$, and postoperative symmetry were significantly associated with good results, with a cut-off value of $38 \%$ of migration percentage and $27^{\circ}$ of acetabular index being predictive. The results were better in combined tenotomies, in diparetic patients more than six years old, and in patients with lower initial values of migration percentage and acetabular index.

\section{Conflicts of interest}

All authors confirm that they did not receive any internal or external fund for the presented work. All authors have no conflicts of interest to declare.

\section{Funding}

No funding was received for the submitted work.

\section{References}

1. Kalen V, Bleck EE (1985) Prevention of spastic paralytic dislocation of the hip. Dev Med Child Neurol 27, 17-24.

2. Cornell MS, Hatrick NC, Boyd R, Baird G, Spencer JD (1997) The hip in children with cerebral palsy. Predicting the outcome of soft tissue surgery. Clin Orthop Relat Res 340, 165-171.

3. Parrott J, Boyd RN, Dobson F, et al. (2002) Hip displacement in spastic cerebral palsy: Repeatability of radiologic measurement. J Pediatr Orthop 22, 660-667.

4. Terjesen T, Lie GD, Hyldmo AA, Knaus A (2005) Adductor tenotomy in spastic cerebral palsy. A long-term follow-up study of 78 patients. Acta Orthop 76, 128-137.
5. Turker RJ, Lee R (2000) Adductor tenotomies in children with quadriplegic cerebral palsy: Lnger term follow-up. J Pediatr Orthop 20, 370-374.

6. Presedo A, Oh CW, Dabney KW, Miller F (2005) Soft-tissue releases to treat spastic hip subluxation in children with cerebral palsy. J Bone Joint Surg Am 87, 832-841.

7. Shore BJ, Yu X, Desai S, Selber P, Wolfe R, Graham HK (2012) Adductor surgery to prevent hip displacement in children with cerebral palsy: The predictive role of the Gross Motor Function Classification System. J Bone Joint Surg Am 94, 326-334.

8. Onimus M, Allamel G, Manzone P, Laurain JM (1991) Prevention of hip dislocation in cerebral palsy by early psoas and adductors tenotomies. J Pediatr Orthop 11, 432-435.

9. Abel MF, Wenger DR, Mubarak SJ, Sutherland DH (1994) Quantitative analysis of hip dysplasia in cerebral palsy: A study of radiographs and 3-D reformatted images. J Pediatr Orthop 14, 283-289.

10. Fucs PMMB, Santili C, Svartman C, Assumpção RMC, Petto DC, Garcia HRP (2006) Fatores preditivos para evolução insatisfatória de quadris instáveis na paralisia cerebral submetidos à reconstrução articular. Acta Ortop Bras 14, 249-252.

11. Soo B, Howard JJ, Boyd RN, et al. (2006) Hip displacement in cerebral palsy. J Bone Joint Surg Am 88, 121-129.

12. Bowen RE, Kehl DK (2006) Radiographic outcome of softtissue surgery for hip subluxation in non-ambulatory children with cerebral palsy. J Pediatr Orthop 15, 109-112.

13. Šponer P, Pellar D, Kucera T, Karpas K (2006) Our approach to the spastic hip subluxation and dislocation in children with cerebral palsy. Act Med 49, 215-218.

14. Hägglund G, Alriksson-Schmidt A, Lauge-Pedersen H, RodbyBousquet E, Wagner P, Westbom L (2014) Prevention of dislocation of the hip in children with cerebral palsy: 20-year results of a population-based prevention programme. Bone Joint J 96-B, 1546-1552.

15. Tönnis D (1976) Normal values of the hip joint for the evaluation of X-rays in children and adults. Clin Orthop Relat Res 119, 39-47.

16. Ha M, Okamoto T, Fukuta $T$, et al (2018) Preoperative radiologic predictors of successful soft tissue release surgery for hip subluxation among cerebral palsy patients: A STROBE compliant study. Medicine (Baltimore) 97, e11847.

17. Schmale GA, Eilert RE, Chang F, Seidel K (2006) High reoperation rates after early treatment of the subluxating hip in children with spastic cerebral palsy. J Pediatr Orthop 26, 617-623.

18. Oh CW, Presedo A, Dabney KW, Miller F (2007) Factors affecting femoral varus osteotomy in cerebral palsy: A longterm result over 10 years. J Pediatr Orthop B 16, 23-30.

19. Macias-Merlo L, Bagur-Calafat C, Girabent-Farrés M, Stuberg WA (2016) Effects of the standing program with hip abduction on hip acetabular development in children with spastic diplegia cerebral palsy. Disabil Rehabil 38, 1075-1081.

20. Ching BH, Khoo TB (2017) Prevalence and predictive factors of hip displacement in children with cerebral palsy at Paediatric Institute Kuala Lampur Hospital. Neurol Asia 22, 243-252. 